\title{
INELASTIC HADRON DIFFRACTION IN HIGH ENERGY ELASTIC SCATTERING OF NUCLEI
}

\author{
A. MAŁECKI AND D. GOC-JAGEO \\ KEN Pedagogical University, Kraków, Poland \\ E-mail: amalecki@ultra.ap.krakow.pl \\ M. PALLOTTA \\ Laboratori Nazionali di Frascati dell'INFN, Italy \\ E-mail: ma.pallotta@tiscalinet.it
}

\begin{abstract}
The rôle of inelastic diffraction in elastic scattering of nuclei is studied in the formalism of diffractive limit. The results obtained for scattering of the $\alpha$-particles on light nuclei show that the nucleonic diffraction is especially important at large momentum transfers where the Glauber model of geometric diffraction fails.
\end{abstract}

\section{Introduction}

Inelastic hadron diffraction refers to quasi-elastic transitions in which the colliding particles conserve their dominant quantum numbers. High energy of collision facilitates to satisfy the condition of coherence thus assuring that interaction does not change the character of the particles.

High energy elastic scattering of hadrons is thus generally built of:

i) elastic diffraction which results from a strong absorption connected to an opening of a variety of inelastic channels and by analogy to classical optics it can be described in terms of geometrical shapes of colliding particles;

ii) inelastic diffraction which appears as a quantum phenomenon related to intrinsic structure of hadrons and has no classical analogy.

It will be shown that elastic (classical or geometrical) diffraction is dominating at low momentum transfers, especially in the region of the forward peak. Instead, the inelastic (quantum or dynamical) diffraction is particularly important at high momentum transfers.

\section{Diffractive transitions}

The most convenient basis for calculating the diffractive amplitude is provided by the experimentally revealed division of inelastic channels into the diffractive and non-diffractive transitions. This means that the space of physical states is decomposable (with respect to the initial state) into subspaces of diffractive $[D]$ and non-diffractive states $[\sim D]$. In other words there exists a unitary

ws-p8-50x6-00: submitted to World Scientific on October 26, 2018 
operator $U$ which is reducible (block-diagonal) in the Hilbert space:

$$
\langle k|U| j\rangle=0 \quad \text { for any }|j\rangle \in[D] \text { and }|k\rangle \in[\sim D] .
$$

Expanding the initial $|i\rangle$ and final $|f\rangle$ states in the basis of $|U j\rangle$ states one obtains the transition amplitude:

$$
T_{f i} \equiv\langle f|T| i\rangle=\sum_{|j\rangle,|k\rangle \in[D]} U_{f k} t_{k j} U_{i j}^{*}
$$

where $U_{f k}=\langle f \mid U k\rangle, \quad t_{k j}=\left\langle k\left|U^{\dagger} T U\right| j\right\rangle \quad$ and $\quad U_{i j}=\langle i \mid U j\rangle$.

In terms of the normal operator $\Lambda \equiv \widehat{1}-U$ this reads:

$$
\begin{array}{r}
T_{f i}=t_{i i} \delta_{f i}-\sum_{|k\rangle} \Lambda_{f k} t_{k i}-\sum_{|j\rangle} t_{f j} \Lambda_{i j}^{*}-\sum_{|j\rangle,|k\rangle} \Lambda_{f k} t_{k j} \Lambda_{k i}^{*} \\
=t_{i} \delta_{f i}-N_{f i}\left(T_{0}\right) \Lambda_{f i} t_{i}-t_{f} \Lambda_{i f}^{*} N_{i f}^{*}\left(T_{0}^{\dagger}\right)+\sum_{|j\rangle \in[D]} N_{f j}\left(T_{0}\right) \Lambda_{f j} t_{j} \Lambda_{i j}^{*}
\end{array}
$$

where $t_{j}=t_{j j}$ are the diagonal matrix elements of $T_{0} \equiv U^{\dagger} T U$ and the undimensional quantities $N_{k j}$ are defined as

$$
N_{k j}\left(T_{0}\right)=\frac{1}{\Lambda_{k j} t_{j j}} \sum_{|l\rangle \in[D]} \Lambda_{k l} t_{l j} .
$$

If the subspace $[D]$ contains a huge number of diffractive states then $N_{k j}=N \rightarrow \infty$ for any pair of states $|k\rangle$ and $|j\rangle$. In fact, since $\Lambda$ is a nonsingular operator its matrix elements change smoothly under the change of diffractive states. This leads to a considerable simplification of Eq.(3):

$$
T_{f i}=t_{i} \delta_{f i}-N\left(\Lambda_{f i} t_{i}+t_{f} \Lambda_{i f}^{*}-\sum_{|j\rangle \in D} \Lambda_{f j} t_{j} \Lambda_{i j}^{*}\right) .
$$

In general, the effect of non-diagonal transitions inside the diffractive subspace $[D]$ gets factorized. In the case of elastic scattering one has:

$$
T_{i i}=t_{i}+N \sum_{|j\rangle \in[D]}\left|\Lambda_{i j}\right|^{2}\left(t_{j}-t_{i}\right)=t_{i}+g_{i} N\left(t_{a v}-t_{i}\right)
$$

where $g_{i}=\sum_{|j\rangle}\left|\Lambda_{i j}\right|^{2}=2 \operatorname{Re} \Lambda_{i i}$ and $t_{a v}=\sum_{|j\rangle}\left|\Lambda_{i j}\right|^{2} t_{j} / g_{i}$ is the average of the diagonal matrix elements $t_{j}$. The expressions of the form $N \Delta t$ where $\Delta t$ represents diversity of $t_{j}$ over the subspace of diffractive states $[D]$ are to be considered in the double diffractive limit [1]: $N \rightarrow \infty, \Delta t \rightarrow 0$ such that $N \Delta t$ is finite. The contribution of inelastic diffraction is thus built as an infinite sum of the infinitesimal contributions from all possible intermediate states belonging to $[D]$. 


\section{Phenomenology of diffractons}

Our numerical analysis of elastic scattering was done in the framework of a model where the diffractive states are built of a two-hadron core (representing the ground state) and some quanta describing diffractive excitations:

$$
|j\rangle=|i\rangle+\left|n ; \vec{b}_{1} \ldots \vec{b}_{n}\right\rangle .
$$

The configurations of these quasi-particles [2] (called diffractons) are specified by a number of constituents and their impact parameters. Thus

$$
\frac{1}{g_{i}} \sum_{|j\rangle \in[D]}\left|\Lambda_{i j}\right|^{2} \ldots=\sum_{n=1}^{\infty} P_{n} \int d^{2} b_{1} \ldots d^{2} b_{n} \prod_{k=1}^{n}\left|\Psi\left(b_{k}\right)\right|^{2} \ldots
$$

where $\left|\Psi\left(b_{k}\right)\right|^{2}$ describe a spatial distribution of diffractons (with respect to the core) and $P_{n}$ are probabilities of their number.

The diagonal elements of $T_{0}$ are assumed to be purely absorptive. In terms of the real profile functions we have:

$$
N\left(t_{j}-t_{i}\right)=i\left(1-\Gamma_{0}\right) \lim _{N \rightarrow \infty, \gamma \rightarrow 0} N \sum_{k=1}^{n} \gamma\left(\vec{b}-\vec{b}_{k}\right)
$$

with $t_{i}=i \Gamma_{0}$ representing the hadronic core and $\gamma^{\prime}$ s corresponding to diffractons. The diffracton model thus explicitly accounts for the geometrical diffraction on an absorbing hadronic bulk and the dynamical diffraction corresponding to intermediate transitions between diffractive states.

For elementary hadron collisions the elastic scattering profile resulting from Eqs. (6), (8) and (9) would read: $\Gamma_{e l}(b)=\Gamma_{0}+\left(1-\Gamma_{0}\right) \Gamma_{n}$. In the case of nucleus-nucleus scattering there are, howewer, two sources of dynamical diffraction. At the nuclear level the diffractive excitations correspond to various configurations of nucleons while the partonic compositness of nucleons gives rise to the subnuclear diffraction. Therefore the elastic profile reads:

$$
\Gamma_{e l}(b)=\Gamma_{0}+\left(1-\Gamma_{0}\right) \Gamma_{N}+\left(1-\Gamma_{0}\right)\left(1-\Gamma_{N}\right) \Gamma_{n}
$$

where $\Gamma_{N}$ and $\Gamma_{n}$ refer to nuclear and nucleonic diffractiveness, respectively.

In our calculations we took the geometrical diffraction profile of two nuclei with $A$ and $B$ nucleons as $\Gamma_{0}=\left(1-\gamma_{A B}\right)^{A B}$ which corresponds to multiple scattering between nucleons frozen in the nuclear ground states. The function $\gamma_{A B}$ and the dynamical profiles $\Gamma_{N}$ and $\Gamma_{n}$ were taken in the Gaussian form: $\left(\sigma / 4 \pi R^{2}\right) \exp \left(-b^{2} / 2 R^{2}\right)$. The parameters of $\gamma_{A B}$ are related to the total nucleon-nucleon cross-section and the radii of the colliding nuclei. The

ws-p8-50x6-00: submitted to World Scientific on October 26, 2018 


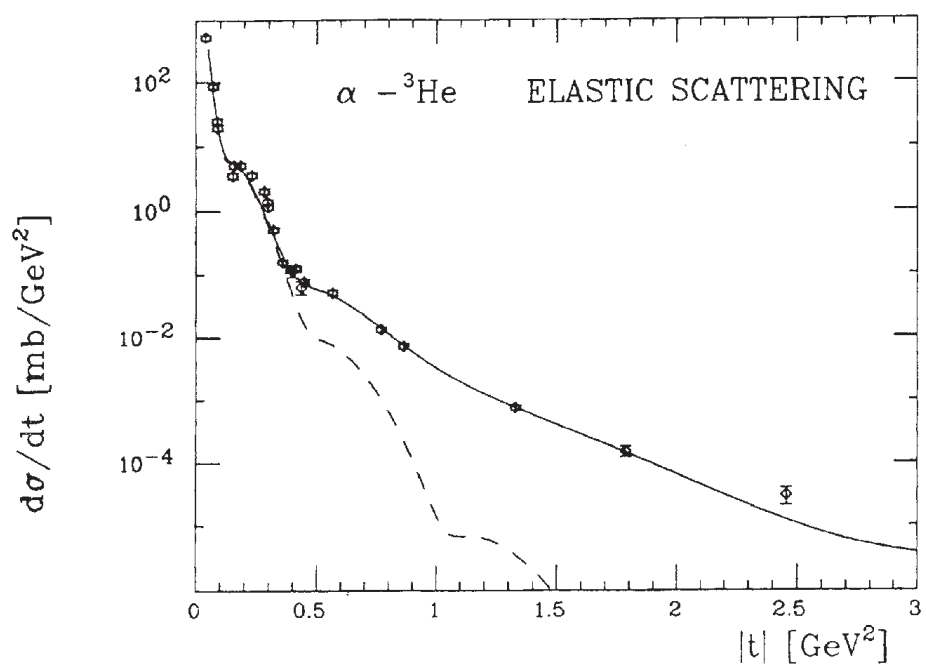

Figure 1. The ${ }^{4} \mathrm{He}-{ }^{3} \mathrm{He}$ elastic cross-section vs. the squared momentum transfer.

parameters of the profiles $\Gamma_{N}$ and $\Gamma_{n}$ were determined from fitting the elastic differential cross-section based on Eq.(10) to experimental data.

In the presented fit to the $\alpha{ }^{-3} \mathrm{He}$ data at $p_{\alpha}=7 \mathrm{GeV} / \mathrm{c}[3]$ we found $R_{N}=0.90 \mathrm{fm}, \sigma_{N}=77 \mathrm{mb}, R_{n}=0.29 \mathrm{fm}$ and $\sigma_{n}=84 \mathrm{mb}$. The comparison with the contribution of the geometrical profile $\Gamma_{0}$ alone (dashed curve) shows that the dynamical terms $\Gamma_{N}$ and $\Gamma_{n}$ become important at high momentum transfers where the Glauber model of multiple scattering fails.

Our analysis reveals the existence of three interaction radii in nucleusnucleus scattering: the external radius $R_{A B}$ describes geometrical diffraction on a black disc, the larger intrinsic radius $R_{N}$ characterises the dynamics of interacting nucleons while the smaller radius $R_{n}$ may be interpreted as the size of partonic clusters inside the nucleons. However, the large value of subnuclear cross-section $\sigma_{n}>\sigma_{N}$ could signal an enhanced population of partons and their deconfinement in the whole nuclear interior.

\section{References}

1. A. Małecki, Phys. Rev. D 54, 3180 (1996).

2. A. Małecki, Phys. Rev. C 44, 1273 (1991).

3. L. Satta et al., Phys. Lett. B 139, 263 (1984). 